\title{
Impact of whey protein isolate coatings containing different antimicrobial agents on sliced bologna-type sausage during refrigerated storage
}

\author{
Selin KALKAN ${ }^{1}$ (D), Zerrin ERGINKAYA ${ }^{2}$
}

\begin{abstract}
In this study, we aimed to determine the possibility of using the edible whey protein isolates coatings containing different antimicrobial agents on the bologna-type sausage slices and the inactivation effects of these coatings against Listeria innocua. For this purpose, edible whey protein isolates (WPI) coatings were prepared to contain essential oils (thyme, coriander, pimento, rosemary, basil), potassium sorbate (PS), sodium benzoate (SB)( $4 \% \mathrm{w} / \mathrm{v})$ and $\mathrm{Nisin}(10.000 \mathrm{IU})$. The antimicrobial effect against L. innocua, sensory characteristics (appearance, color, odor, texture, flavor and overall acceptability) and physical (color) and chemical ( $\mathrm{pH}$ and water activity) properties of bologna slices coated WPI coatings were determined for during the 35-day period in refrigerated conditions $\left(4 \pm 1^{\circ} \mathrm{C}\right)$. In conclusion, it was determined that the most effective coatings for $L$. innocua inactivation were PS, SB, and pimento essential oil, approximately 4.69, 4.45 and $4.16 \log$ CFU/g, respectively. The most effective coating on the $\mathrm{pH}$ values (5.90-6.04) was established to be WPI coatings containing Nisin. It has been found that WPI coatings containing thyme essential oil provided the lowest water activity values with $\mathbf{0 . 9 4 5}$. In addition, it was possible to say that the most favored type of coating for sausage samples was Nisin-WPI coatings with 7.63- 8.33 sensory scores.
\end{abstract}

Keywords: edible coatings; antimicrobial; Listeria innocua; whey protein isolate; food packaging.

Practical Application: Post-processing contamination in ready to eat meat products with Listeria spp. became a major concern for the meat processing industry and an important food safety issue. The results of this study confirmed that edible antimicrobial whey protein isolate coatings with enriched different antimicrobial agents could reduce the growth rate of L. innocua in sliced emulsion-type sausage. These coatings can lead to better quality preservation of the emulsion bologna-type sausages stored at low temperature and can extend their shelf life.

\section{Introduction}

In recent years, due to the increased interest in minimally processed foods depending on consumer demand, use of new technologies and approaches started in the packaging industry. The active packaging technique is the most prominent of these technologies (Erginkaya et al., 2014). Antimicrobial packaging, as an active packaging technique, is a suitable protection method, especially for raw meat, processed meat products, poultry, dairy products, and seafood (Suppakul et al., 2003; Karagöz \& Candoğan, 2007). Controlled release of antimicrobial compounds carried out on these systems can inhibit both the initial microorganisms and the current microbial growth during storage. These systems form a barrier mechanism for pathogens and/or spoilage microorganism for ensuring food safety (Cooksey, 2005). Traditionally, the antimicrobial effect of edible coatings has been obtained by incorporating in their structure antimicrobial agents, such as enzymes, polysaccharides, bacteriocins and more recently herbs, spices and essential oils (Appendini \& Hotchkiss, 2002; Pranoto et al., 2005; Tajkarimi et al., 2010). The formation of edible films and coatings from whey proteins can increase the utilization of whey, improve the nutritional value of foods and prolong shelf life (Ozdemir \& Floros, 2008).
Bologna sausage, a type of minimally cooked meat product originating in Italy, is made with finely ground, cured beef and pork, with seasonings similar to frankfurters (Davies et al., 1999). In many studies have shown that post-processing contamination in ready-to-eat meat products are the main source of some pathogens and this risk is increased by slicing (Cağri et al., 2002; Zhu et al., 2005). In particular, L. monocytogenesis seen as a major contaminant of food poisonings originating from ready-to-eat meat products as a common surface contaminant. Listeriosis which caused by Listeria monocytogenes has been recognized as a serious public health hazard, with high mortality rates in susceptible individuals, such as the elderly and immunocompromised (Barancelli et al., 2014). Because of this, studies have focused on the elucidation of the microbial ecology of foods in relation to this bacterium (Fernandes et al., 2013). Listeria innocua has been an important surface contaminant in terms of food microbiology since the presence of Listeria innocua in food products would represent a possible presence of Listeria monocytogenes (Sheridan et al., 1997; Buchrieser et al., 2003). Appropriateness of using L. innocua as surrogates for $L$. monocytogenes has been previously reported (Pietrysiak \& Ganjyal, 2018; Kim et al., 2018). While antimicrobial coatings reduce to surface contaminants such as Listeria spp., the consumers in terms of their sensory properties should like them. 
In this context, the use of descriptive sensory methodologies applied to consumers seems to be a valuable alternative due to the economic advantages and the possibility of generating a preliminary vocabulary (Santos et al., 2015).

Methods involving the use of antimicrobial agents with film and coatings are in the developmental stage. The antimicrobial packaging systems studies, which developing taken into account technological suitability, consumer acceptability and food safety are required for ready-to-eat processed meat products (Karagöz \& Candoğan, 2007). The usage of this type of whey protein film may be limiting in products where the absence of lactose is a required priority. However, processed meat products use mostly whey protein to modify the overall technological and sensory characteristics of a meat system such as water holding capacity, fat holding capacity and texture properties so it was a justified matrix to use (Odila Pereira et al., 2018). As a by-product of the cheese industry, whey is a highly promising food market due to its bioactive peptides, antioxidants and health benefits associated with essential amino acids. Whey protein is a source of essential amino acids, bioactive peptides, antioxidants and immunopotentiators that provide a number of functions in the human body. The bioactive compounds of whey have elimination of free radicals, anti-inflammatory, antitumor, antiobesity and antidiabetic properties, acts on muscle biosynthesis, osteoprotection and radioprotection (Amaral et al., 2018; Coutinho et al., 2019; Silveira et al., 2019). Whey proteins are quite suitable for use as edible films and coatings with all these beneficial properties.

This study aimed to investigate the impact of whey protein isolates coatings containing different antimicrobial agents on the bologna-type sausage and the inactivation effects of these antimicrobial coating materials against Listeria innocua, during the storage period.

\section{Material and methods}

\subsection{Material}

Bologna-type sausage [a kind of emulsion meat product] baton-type samples, produced by Pinar Integrated Meat and Flour Industry (Kemalpaşa, İzmir, Turkey), were used in the experiments. Bologna-type sausage samples were obtained from the local market and brought to the laboratory under suitable conditions (at $4{ }^{\circ} \mathrm{C}$ in original packaging). Whey protein isolate (BIPRO Whey Protein Isolate) from Davisco Foods International. Inc.-USA. Hardline Nutrition, obtained from Turkey. The thyme, basil, coriander, rosemary and pimento essential oils, Nisin, sodium benzoate, potassium sorbate, wax and antifoam (silicon antifoam) were obtained from Sigma Chemical (St. Louis, Mo., USA). Glycerol (Merck; Darmstadt, Germany) was used as the plasticizer. Listeria innocua (ATCC 33090) was used as a bacterial culture in the study.

\subsection{Preparation of antimicrobial coating}

The whey protein isolates (WPI; 5\% w/v) and glycerol $(2 \% \mathrm{w} / \mathrm{v})$ were dissolved in $100 \mathrm{~mL}$ distilled water containing $0.04 \% \mathrm{CaCl}_{2}(\mathrm{w} / \mathrm{h})$. The $\mathrm{pH}$ of the solution was adjusted to 8 using $1 \mathrm{M} \mathrm{NaOH}$ and then solution heated with stirring at $90^{\circ} \mathrm{C}$ for 30 minutes. $0.1 \mathrm{~mL}$ of anti-foaming agent was added while mixing. During the last 5 minutes of heating, the wax $(0.4 \% \mathrm{w} / \mathrm{v})$ was slowly added. Homogenization of the solution for 2 minutes at 22,000 rpm was achieved by using a homogenizer. After the filtration of the solution was cooled to $23 \pm 2{ }^{\circ} \mathrm{C}$ and $10.000 \mathrm{IU}$ of Nisin was added to the cooled solution. Thyme, rosemary, basil, pimento and coriander essential oils were added to the coating solution at the rate of $4 \%(\mathrm{w} / \mathrm{v})$ for each coating solution. Essential oils were primarily dissolved in Tween 80 ( $0.250 \mathrm{~g}$ Tween 80 / g essential oil) in order to distribute the essential oils more homogeneously in solution. The potassium sorbate and sodium benzoate were added to the coating solution at the rate of $4 \%$ (w/v) (Cağri et al., 2002; Sarıkuş, 2006; Cao-Hoang et al., 2010).

\subsection{Microorganism inoculation, coating and storage}

Bologna-type sausage samples were sterilized for 30 minutes under UV light in a sterile cabin after being cut into slices of approximately 10 grams (8 $\mathrm{cm}$ in diameter, $4 \mathrm{~mm}$ thickness) with a sterile knife. $L$. innocua concentration was adjusted to $10^{10} \mathrm{CFU} / \mathrm{mL}$ by incubating at $35^{\circ} \mathrm{C}$ in Nutrient Broth medium. After incubation, cultures were transferred to the centrifuge tube at aseptic conditions. Supernatant was removed after centrifugation at $5000 \mathrm{rpm}$ for 30 minutes. Subsequently, the cell pellet was washed twice with $5 \mathrm{~mL}$ of peptone water $(0.1 \% \mathrm{w} / \mathrm{v}) .100 \mu \mathrm{L}$ of bacterial suspension was transferred from the dilutions prepared from the culture suspended in $5 \mathrm{~mL}$ of peptone water and $500 \mathrm{~mL}$ solution were prepared. Bologna-type sausage slices were left with these solutions for 10 minutes. At the end of this period, the Bologna-type sausage slices taken to sterile petri dishes for absorption of the solution for approximately 30 minutes. The final inoculum level is set to be $10^{6} \mathrm{CFU} / \mathrm{g}$ for each Bologna-type sausage slice (Ku et al., 2008; Tiryaki, 2008; Ye et al., 2008). Then, Bologna-type sausage slices were coated with antimicrobial WPI coating solutions at different contents for 2 minutes. The coating process was repeated one more time. The post-coating, drying was carried out by hanging the Bologna-type sausage samples at $50 \% \mathrm{RH}$ at $25^{\circ} \mathrm{C}$. The excess of liquid in the sliced Bologna-type sausage samples was drained for around $30 \mathrm{~s}$, and thereafter it was packaged (three slices of Bologna-type sausage samples in each package) under usual conditions, i.e. vacuum packaging. After the coating with the film solution, the inoculation of L. innocua concentration was adjusted to $10^{6} \log \mathrm{CFU} / \mathrm{g}$ for each samples. Packages were stored at $4{ }^{\circ} \mathrm{C}$ for 35 days and at each sampling point $(1,7,14,21,28$ and 35 days), all packages of each condition were evaluated.

\subsection{Determination of antimicrobial effects of coatings against Listeria innocua}

Antimicrobial WPI coating applied Bologna-type sausage samples were analyzed for the determinate of L. innocua inoculation levels on days 1, 7, 14, 21, 28 and 35 of the storage. For this purpose, 10 grams Bologna-type sausage slices were added to $90 \mathrm{~mL}$ of $0.1 \%$ sterile peptone water ( $\mathrm{pH} 7.2$ ). Samples were homogenized with Stomacher. The analyses were carried out in 2 parallel and 3 replicates in accordance with the FDA Bacteriological Analytical Manual (Andrews et al., 1995). Serial dilutions were prepared from the homogenized samples. The prepared dilutions were planted to Oxford Listeria Selective 
Agar (Merck) medium. The counts of all black colonies, which have around the shadows, were made at the end of the 24-hour incubation at $35^{\circ} \mathrm{C}$ (Tomé et al., 2008).

\subsection{Chemical and physical analysis of sliced Bologna-type sausage}

The $\mathrm{pH}$ values of the samples were determined with a pH meter (Ohaus Starter 3000 Parsippany, NJ 07054, USA). The water activities of the samples were measured at $25{ }^{\circ} \mathrm{C}$ using a AquaLab 4TE (Pullman, Washington, USD). Color properties, $L^{*}$ (openness), $a^{*}$ (red-green) and $b^{*}$ (yellow-blue), of antimicrobial WPI covered Bologna-type sausage samples were measured with CR-410 colorimeter (Minolta Chroma, Osaka, Japan) on the white standard surface during the storage period. The color change $\left(\Delta \mathrm{E}^{\star}\right)$ of the Bologna-type sausage samples was calculated by using the equation (Equation 1) (Zinoviadou et al., 2009; Pires et al., 2011; Bahram et al., 2013).

$\Delta E^{*}=\left(\left(L_{o}^{*}-L^{*}\right)^{2}+\left(a_{o}^{*}-a^{*}\right)^{2}+\left(b_{o}^{*}-b^{*}\right)^{2}\right)^{1 / 2}$

As seen in the equation, $L_{0}^{*}, a_{0}^{*}$ and $b_{0}^{*}$ values are the color parameters of the standard surface, while $L^{*}, a^{*}$ and $b^{*}$ values are the color parameters of the samples.

\subsection{Sensory analysis}

Sensory analysis was carried out in which only on L. innocua non-inoculated and covered with antimicrobial-WPI bologna-type sausage samples. Stored samples were assessed by 8 panelists using a sensory rating scale of 1 (poor) to 9 (excellent) for some sensory parameters such as general appearance, color, odor, texture, flavor and overall acceptability. The assessors were instructed to rinse their mouths with distilled water between samples to avoid carry-over effect. The sessions were held at the Laboratory of Food Engineering of Çukurova University (Horita et al., 2017; Vidal et al., 2019).

\subsection{Statistical analysis}

Statistical analyzes were performed using the "Windows SPPS 20.0 software" statistical package program (SPSS Inc., Chiago, IL, USA). One-way analysis of variance (ANOVA) was used to compare differences in significance between tests and Duncan's multiple comparison test was used to compare differences between groups $(p<0.01 ; p<0.05)$ (Royo et al., 2010).

\section{Results and discussion}

\subsection{Antimicrobial analysis}

The effects of antimicrobial WPI coating against $L$. innocua on bologna-type sausage samples stored in refrigerator conditions are shown in Figure 1 and Figure 2.

As shown in Figure 1 and Figure 2, L. innocua concentration decreased compared to the increasing storage days. This result was statistically significant for all WPI coatings containing antimicrobial agent $(\mathrm{p}<0.01)$. As a result of the statistical analyzes, the type of coating used and the storage time were found to be important in the inactivation of L. innocua WPI coatings containing potassium sorbate, sodium benzoate and pimento essential oil provided the most effective inactivation against $L$. innосиа. WPI coatings containing Nisin, thyme, basil, coriander and rosemary essential oils showed the antimicrobial effect against $L$. innocua, respectively. $L$. innocua inactivation approximate was $4.69 \mathrm{log}$ CFU/g by using PS-WPI coatings compared to the control group. Similarly, the inactivation of L. innocua was about $4.45 \mathrm{log}$ CFU/g by using SB-WPI coatings. Another important coating, Nisin-WPI, provided the inactivation of approximately $3.12 \log \mathrm{CFU} / \mathrm{g}$ L. innocua. As expected, there

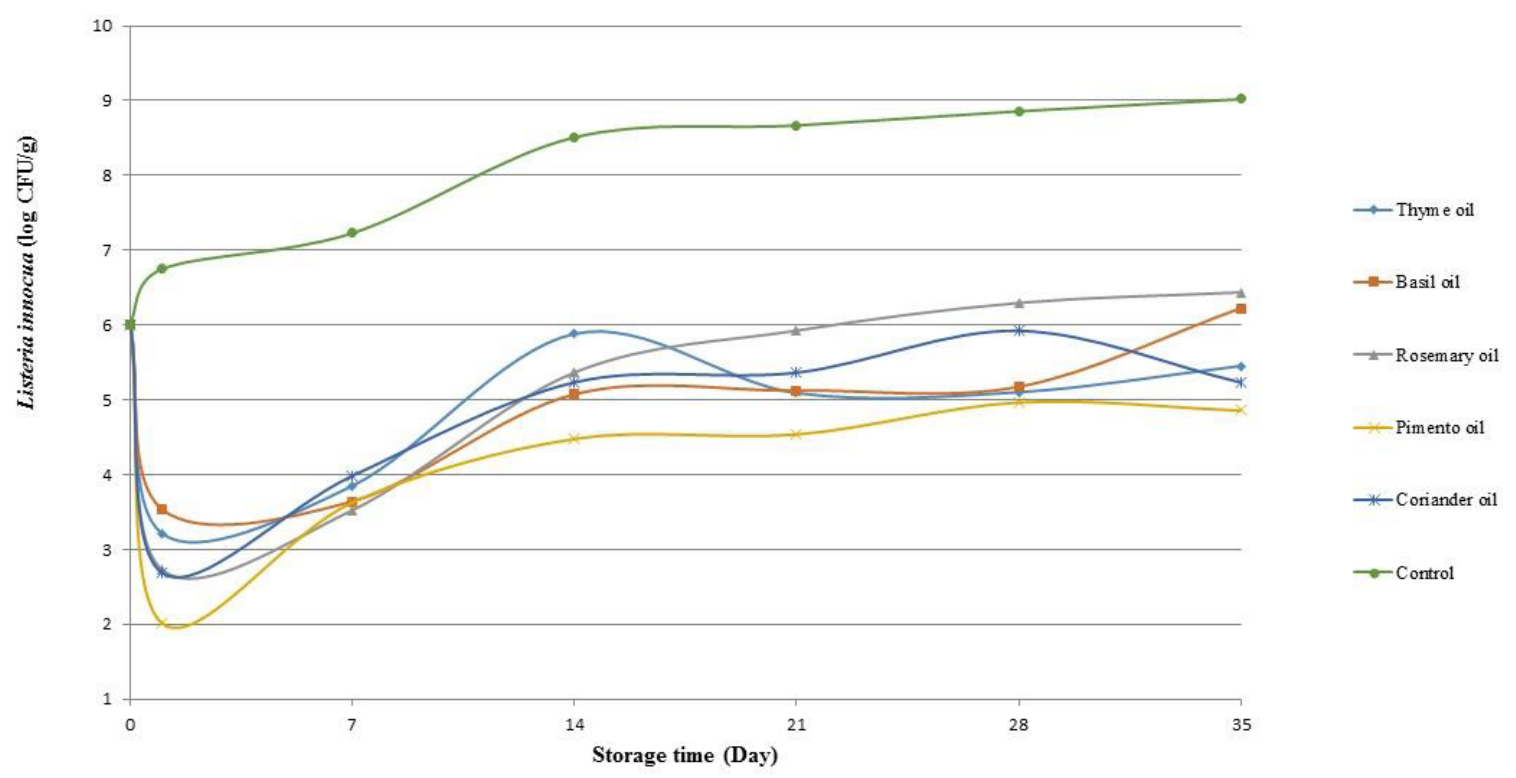

Figure 1. L. innocua inactivation on Bologna-type sausage slices coated with WPI coating solution containing essential oils (log CFU/g). 


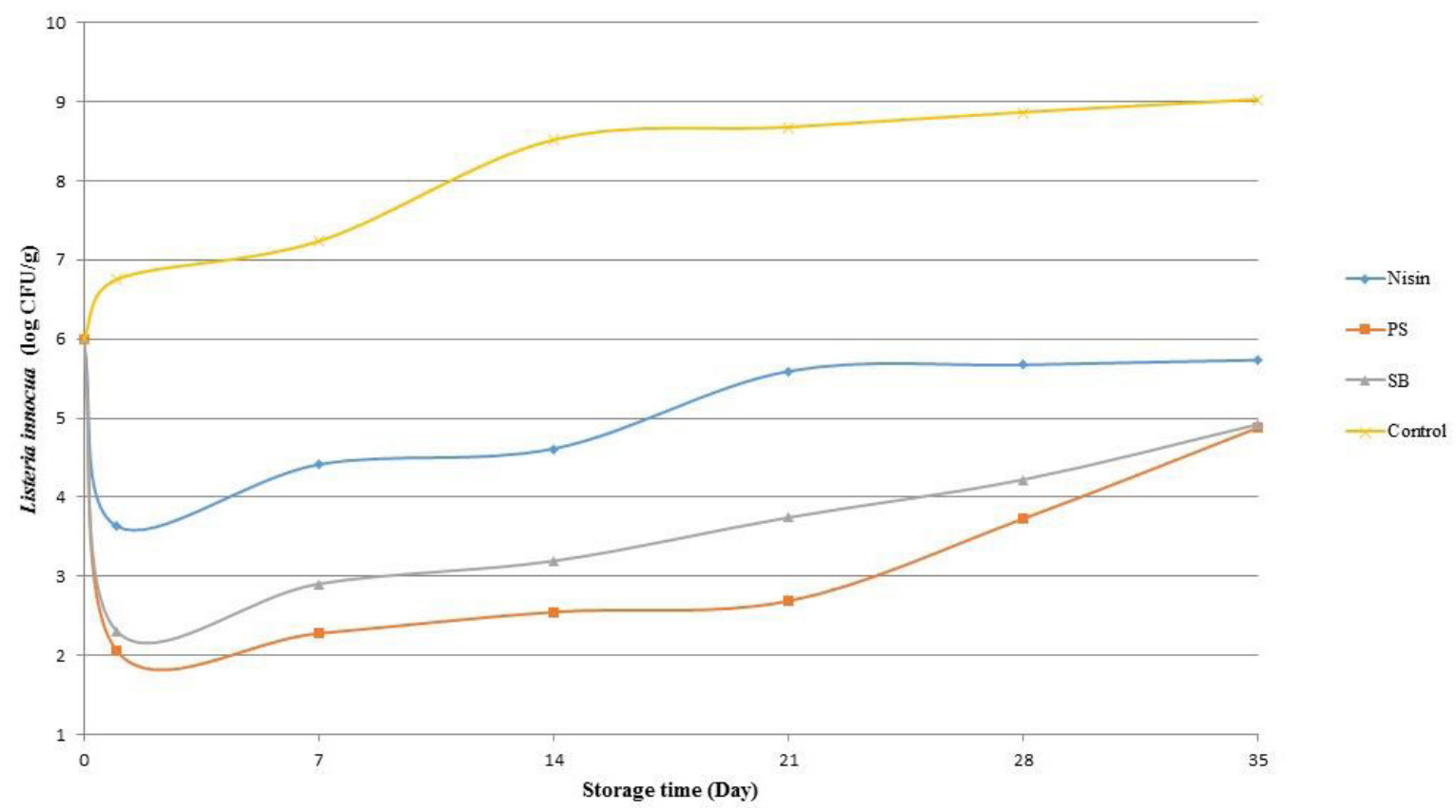

Figure 2. L. innocua inactivation on Bologna-type sausage slices coated with WPI coating solution containing nisin, potassium sorbate and sodium benzoate $(\log \mathrm{CFU} / \mathrm{g})$.

was a logarithmic increase in the number of $L$. innocua and it has reached approximately 1.5 times at the end of storage period $(\mathrm{p}<0.01)$.

When the effect of the storage time on the number of L. innocua was examined, the 1, 7, 14 and 21 of the storage days determined the maximum reduction. L. innocua counts increased at 28 and 35 days of the storage $(p<0.01)$. Sorbate, benzoate, and propionate are generally recognized as safe additives that are traditionally used to control mold growth in a variety of food products (Food and Drug Administration, 2006). These antimycotic agents have also been shown to inhibit growth of gram-positive bacterial pathogens, such as Clostridium botulinum, Staphylococcus aureus, and Listeria monocytogenes in media, and in meat systems (Glass et al., 2007). Previous studies revealed that $0.1 \%$ or more of total potassium sorbate, propionic acid, or benzoic acid, alone or in combination, will prevent the growth of L. monocytogenes in turkey and frankfurter slurries when stored at $4^{\circ} \mathrm{C}$ for 4 weeks (Glass et al., 2004). The results of these experiments also suggested that benzoate had greater antilisterial activity than propionate or sorbate on a weight basis. However, exclusive use of benzoate has limitations. High levels of benzoate may adversely affect the flavor of the finished product, although this problem may be lessened by the use of benzoate in combination with another effective antimicrobial agent (Glass et al., 2007). Furthermore, if benzoate is included in a mixture, current U.S. Food and Drug Administration regulations limit the total antimycotic agents to $0.1 \%$ (Food and Drug Administration, 2006). In addition, several studies have shown the bactericidal effect of nisin and pediocins against L. monocytogenes in raw and heated as well as in fermented and unfermented meat products (Murray \& Richard, 1997). Similarly, nisin shows antimicrobial activity against L. innocua. Similar to the results of the study, Theivendran et al. (2006) reported that L. monocytogenes concentration was decreased $2.3 \mathrm{log} \mathrm{CFU} / \mathrm{g}$ in turkey sausages covered with WPI containing Nisin. Cağri et al. (2002) have applied WPI coating containing p-aminobenzoic acid and sorbic acid to Bologna-type sausage slices stored at refrigerator temperature and they provided inactivation of L. monocytogenes. Similarly, Ramos et al. (2012) stated that bacteriostatic effect was obtained except for coatings containing rosemary extract, and a decrease of about 2.5 logarithms of bacterial concentration was detected on days 5 and 7 of storage. In our study, it was determined that WIP coatings containing rosemary essential oil were found to be less effective against L. innocua. In the light of the data obtained from our study, the antimicrobial effect against L. innocua with the antimicrobial properties of WPI coatings will provide a similar effect against L. monocytogenes, which is common surface contamination in heat-treated meat products. Thus, both product safety and public health protection will be ensured.

\subsection{Chemical and physical properties of sliced Bologna-type sausage samples}

The changes in the $\mathrm{pH}$, water activity $\left(\mathrm{a}_{\mathrm{w}}\right)$ values and color properties of the bologna-type sausage samples coated with the antimicrobial WPI and stored under refrigerated conditions represented in Table 1, Table 2 and Table 3. As seen in Table 1, pH values were decreased in samples applied PASP coating according to increased storage days. These results were insignificant statistically for PASP coatings containing potassium sorbate, sodium benzoate ( $4 \% \mathrm{w} / \mathrm{v})$, nisin (10.000 IU) and control group (without any antimicrobials). However, they were found to be important statistically for other samples, which applied antimicrobial coatings. 
Table 1. $\mathrm{pH}$ values of Bologna-type sausage samples covered with antimicrobial WIP coatings.

\begin{tabular}{|c|c|c|c|c|c|c|}
\hline \multirow{2}{*}{$\begin{array}{c}\text { Antimicrobial WIP } \\
\text { coatings }^{*}\end{array}$} & \multicolumn{6}{|c|}{ Storage time (days) } \\
\hline & 1 & 7 & 14 & 21 & 28 & 35 \\
\hline Tyhmeoil & $6.54 \pm 0.02^{\mathrm{B}}$ & $6.50 \pm 0.03^{\text {B }}$ & $6.32 \pm 0.04^{\mathrm{A}}$ & $6.26 \pm 0.03^{\mathrm{A}}$ & $6.30 \pm 0.03^{\mathrm{A}}$ & $6.30 \pm 0.01^{\mathrm{A}}$ \\
\hline Basil oil & $6.48 \pm 0.02^{\mathrm{B}}$ & $6.44 \pm 0.02^{\mathrm{B}}$ & $6.12 \pm 0.03^{\mathrm{A}}$ & $6.10 \pm 0.05^{\mathrm{A}}$ & $6.18 \pm 0.01^{\mathrm{A}}$ & $6.02 \pm 0.12^{\mathrm{A}}$ \\
\hline Rosemaryoil & $6.51 \pm 0.01^{\mathrm{B}}$ & $6.55 \pm 0.01^{\mathrm{B}}$ & $6.24 \pm 0.03^{\mathrm{A}}$ & $6.31 \pm 0.03^{\mathrm{A}}$ & $6.31 \pm 0.04^{\mathrm{A}}$ & $6.28 \pm 0.01^{\mathrm{A}}$ \\
\hline Corianderoil & $6.48 \pm 0.02^{\mathrm{C}}$ & $6.46 \pm 0.02^{\mathrm{C}}$ & $6.33 \pm 0.03^{\text {В }}$ & $6.28 \pm 0.00^{\mathrm{AB}}$ & $6.24 \pm 0.03^{\mathrm{A}}$ & $6.29 \pm 0.04^{\mathrm{AI}}$ \\
\hline Pimentooil & $6.41 \pm 0.03^{\mathrm{CD}}$ & $6.50 \pm 0.03^{\mathrm{D}}$ & $6.31 \pm 0.03^{\mathrm{BC}}$ & $6.27 \pm 0.03^{\mathrm{AB}}$ & $6.19 \pm 0.05^{\mathrm{A}}$ & $6.16 \pm 0.03^{\mathrm{A}}$ \\
\hline Nisin & $5.94 \pm 0.09^{\mathrm{A}}$ & $6.04 \pm 0.00^{\mathrm{A}}$ & $5.95 \pm 0.04^{\mathrm{A}}$ & $5.91 \pm 0.05^{\mathrm{A}}$ & $5.90 \pm 0.02^{\mathrm{A}}$ & $5.93 \pm 0.03^{\mathrm{A}}$ \\
\hline PS & $6.40 \pm 0.02^{\mathrm{A}}$ & $6.49 \pm 0.07^{\mathrm{A}}$ & $6.42 \pm 0.02^{\mathrm{A}}$ & $6.40 \pm 0.02^{\mathrm{A}}$ & $6.52 \pm 0.03^{\mathrm{A}}$ & $6.40 \pm 0.04^{\mathrm{A}}$ \\
\hline SB & $6.53 \pm 0.04^{\mathrm{A}}$ & $6.53 \pm 0.05^{\mathrm{A}}$ & $6.57 \pm 0.02^{\mathrm{A}}$ & $6.57 \pm 0.06^{\mathrm{A}}$ & $6.50 \pm 0.07^{\mathrm{A}}$ & $6.42 \pm 0.04^{\mathrm{A}}$ \\
\hline Control & $6.55 \pm 0.01^{\mathrm{A}}$ & $6.64 \pm 0.02^{\mathrm{A}}$ & $6.28 \pm 0.03^{\mathrm{B}}$ & $6.27 \pm 0.08^{\mathrm{B}}$ & $6.31 \pm 0.10^{\mathrm{B}}$ & $6.32 \pm 0.06^{\mathrm{B}}$ \\
\hline
\end{tabular}

${ }^{*}$ Mean and standard deviation; on the table, the same letters in lines and columns Express no difference statistically $(p>0.01)$, different letters express a difference statistically ( $\left.\mathrm{p}<0.05\right)$.

Table 2. Water activity $\left(\mathrm{a}_{\mathrm{w}}\right)$ values of Bologna-type sausage samples covered with antimicrobial WIP coatings.

\begin{tabular}{|c|c|c|c|c|c|c|}
\hline \multirow{2}{*}{$\begin{array}{l}\text { Antimicrobial WIP } \\
\text { coatings }^{*}\end{array}$} & \multicolumn{6}{|c|}{ Storage time (days) } \\
\hline & 1 & 7 & 14 & 21 & 28 & 35 \\
\hline Tyhmeoil & $0.930 \pm 0.007^{\mathrm{A}}$ & $0.946 \pm 0.008^{\mathrm{B}}$ & $0.947 \pm 0.002^{\mathrm{B}}$ & $0.943 \pm 0.005^{\mathrm{AB}}$ & $0.947 \pm 0.000^{\mathrm{B}}$ & $0.953 \pm 0.002^{\mathrm{B}}$ \\
\hline Basil oil & $0.940 \pm 0.001^{\mathrm{A}}$ & $0.947 \pm 0.001^{\mathrm{B}}$ & $0.941 \pm 0.001^{\mathrm{A}}$ & $0.952 \pm 0.001^{\mathrm{BC}}$ & $0.949 \pm 0.000^{\mathrm{B}}$ & $0.955 \pm 0.003$ \\
\hline Rosemaryoil & $0.945 \pm 0.004^{\mathrm{AB}}$ & $0.953 \pm 0.005^{\mathrm{C}}$ & $0.942 \pm 0.002^{\mathrm{A}}$ & $0.950 \pm 0.001^{\mathrm{AB}}$ & $0.950 \pm 0.001^{\mathrm{AB}}$ & $0.951 \pm 0.001$ \\
\hline Corianderoil & $0.939 \pm 0.002^{\mathrm{A}}$ & $0.946 \pm 0.001^{\mathrm{B}}$ & $0.940 \pm 0.001^{\mathrm{A}}$ & $0.952 \pm 0.002^{\mathrm{C}}$ & $0.950 \pm 0.001^{\mathrm{BC}}$ & $0.951 \pm 0.001^{\mathrm{C}}$ \\
\hline Pimentooil & $0.941 \pm 0.002^{\mathrm{A}}$ & $0.945 \pm 0.004^{\mathrm{AB}}$ & $0.941 \pm 0.001^{\mathrm{A}}$ & $0.952 \pm 0.001^{\mathrm{BC}}$ & $0.951 \pm 0.001^{\mathrm{BC}}$ & $0.954 \pm 0.002^{C}$ \\
\hline Nisin & $0.942 \pm 0.002^{\mathrm{A}}$ & $0.952 \pm 0.010^{\mathrm{A}}$ & $0.941 \pm 0.001^{\mathrm{A}}$ & $0.951 \pm 0.002^{\mathrm{A}}$ & $0.953 \pm 0.003^{\mathrm{A}}$ & $0.952 \pm 0.002^{\mathrm{A}}$ \\
\hline PS & $0.945 \pm 0.003^{\mathrm{AB}}$ & $0.945 \pm 0.003^{\mathrm{AB}}$ & $0.941 \pm 0.001^{\mathrm{A}}$ & $0.951 \pm 0.001^{\mathrm{BC}}$ & $0.951 \pm 0.002^{\mathrm{BC}}$ & $0.954 \pm 0.002$ \\
\hline SB & $0.941 \pm 0.002^{\mathrm{A}}$ & $0.945 \pm 0.004^{\mathrm{AB}}$ & $0.942 \pm 0.002^{\mathrm{A}}$ & $0.952 \pm 0.001^{\mathrm{BC}}$ & $0.951 \pm 0.002^{\mathrm{BC}}$ & $0.954 \pm 0.002$ \\
\hline Control & $0.942 \pm 0.006^{\mathrm{AB}}$ & $0.942 \pm 0.007^{\mathrm{A}}$ & $0.953 \pm 0.002^{\mathrm{AB}}$ & $0.953 \pm 0.001^{\mathrm{AB}}$ & $0.957 \pm 0.001^{\mathrm{AB}}$ & $0.959 \pm 0.007^{\mathrm{B}}$ \\
\hline
\end{tabular}

${ }^{*}$ Mean and standard deviation; on the table, the same letters in lines and columns Express no difference statistically $(p>0.01)$, different letters express a difference statistically ( $\left.\mathrm{p}<0.05\right)$.

Table 3. Color properties of Bologna-type sausage slices coated with WPI solution containing different antimicrobials during storage.

\begin{tabular}{|c|c|c|c|c|c|c|c|c|c|}
\hline \multirow{2}{*}{$\begin{array}{c}\text { Color } \\
\text { parameters / } \\
\text { Time (days) }\end{array}$} & \multicolumn{9}{|c|}{ Antimicrobial WPI Coatings } \\
\hline & Control & Tyhmeoil & Basil oil & Corianderoil & Pimentooil & Rosemaryoil & PS & SB & Nisin \\
\hline \multicolumn{10}{|l|}{$L^{*}$} \\
\hline 1 & $52.02 \pm 0.07^{\mathrm{A}}$ & $54.54 \pm 0.29^{\mathrm{A}}$ & $52.40 \pm 0.35^{\mathrm{A}}$ & $57.27 \pm 0.63^{\mathrm{A}}$ & $58.91 \pm 0.07^{\mathrm{A}}$ & $57.37 \pm 0.31^{\mathrm{A}}$ & $55.16 \pm 0.51^{\mathrm{A}}$ & $53.90 \pm 0.12^{\mathrm{AB}}$ & $58.92 \pm 0.11^{\mathrm{A}}$ \\
\hline 7 & $52.68 \pm 0.28^{\mathrm{A}}$ & $55.42 \pm 0.39^{\mathrm{A}}$ & $52.59 \pm 0.14^{\mathrm{A}}$ & $57.88 \pm 0.32^{\mathrm{A}}$ & $57.04 \pm 1.55^{\mathrm{A}}$ & $57.77 \pm 0.33^{\mathrm{A}}$ & $55.09 \pm 0.30^{A}$ & $54.55 \pm 0.56^{\mathrm{A}}$ & $58.09 \pm 0.24^{\mathrm{A}}$ \\
\hline 14 & $53.30 \pm 1.51^{\mathrm{A}}$ & $54.40 \pm 1.23^{\mathrm{A}}$ & $52.85 \pm 0.77^{\mathrm{A}}$ & $56.39 \pm 0.42^{\mathrm{A}}$ & $58.00 \pm 0.13^{\mathrm{A}}$ & $57.09 \pm 0.92^{\mathrm{A}}$ & $55.97 \pm 0.46^{\mathrm{A}}$ & $53.83 \pm 0.71^{\mathrm{AB}}$ & $56.93 \pm 0.42^{\mathrm{A}}$ \\
\hline 21 & $53.18 \pm 0.88^{\mathrm{A}}$ & $52.93 \pm 0.80^{\mathrm{AB}}$ & $53.54 \pm 0.25^{\mathrm{A}}$ & $55.94 \pm 0.79^{\mathrm{A}}$ & $58.00 \pm 0.23^{\mathrm{A}}$ & $57.70 \pm 0.11^{\mathrm{A}}$ & $56.79 \pm 0.05^{\mathrm{A}}$ & $54.61 \pm 0.86^{\mathrm{A}}$ & $57.09 \pm 0.97^{\mathrm{A}}$ \\
\hline 28 & $55.74 \pm 0.48^{\mathrm{A}}$ & $55.74 \pm 0.48^{\mathrm{A}}$ & $51.01 \pm 0.29^{\mathrm{A}}$ & $57.23 \pm 0.32^{\mathrm{A}}$ & $57.05 \pm 1.38^{\mathrm{A}}$ & $58.33 \pm 0.41^{\mathrm{A}}$ & $55.74 \pm 0.48^{\mathrm{A}}$ & $51.01 \pm 0.29^{\mathrm{B}}$ & $57.74 \pm 0.50^{\mathrm{A}}$ \\
\hline 35 & $54.78 \pm 1.97^{\mathrm{A}}$ & $49.88 \pm 0.55^{\mathrm{B}}$ & $52.21 \pm 0.48^{\mathrm{A}}$ & $56.96 \pm 0.26^{\mathrm{A}}$ & $57.79 \pm 0.31^{\mathrm{A}}$ & $56.18 \pm 0.86^{\mathrm{A}}$ & $56.49 \pm 0.13^{\mathrm{A}}$ & $55.60 \pm 0.39^{\mathrm{A}}$ & $57.21 \pm 0.24^{\mathrm{A}}$ \\
\hline \multicolumn{10}{|l|}{$a^{*}$} \\
\hline 1 & $22.56 \pm 0.34^{\mathrm{A}}$ & $22.13 \pm 0.15^{\mathrm{AB}}$ & $22.18 \pm 0.17^{\mathrm{D}}$ & $20.40 \pm 0.24^{\mathrm{A}}$ & $20.87 \pm 0.56^{\mathrm{A}}$ & $21.71 \pm 0.19^{\AA}$ & $22.44 \pm 0.18^{\mathrm{A}}$ & $22.57 \pm 0.23^{\mathrm{AB}}$ & $21.88 \pm 0.22^{\mathrm{A}}$ \\
\hline 7 & $23.82 \pm 0.24^{\mathrm{A}}$ & $22.41 \pm 0.11^{\mathrm{A}}$ & $22.27 \pm 0.34^{\mathrm{A}}$ & $21.08 \pm 0.44^{\mathrm{A}}$ & $20.50 \pm 0.39^{\mathrm{A}}$ & $21.05 \pm 0.17^{\mathrm{AB}}$ & $21.95 \pm 0.17^{\mathrm{A}}$ & $22.80 \pm 0.10^{\mathrm{A}}$ & $21.82 \pm 0.16^{\mathrm{A}}$ \\
\hline 14 & $22.01 \pm 0.95^{\mathrm{A}}$ & $21.27 \pm 0.55^{\mathrm{AB}}$ & $20.86 \pm 0.16^{\mathrm{B}}$ & $19.91 \pm 0.28^{\mathrm{AB}}$ & $20.40 \pm 0.15^{\mathrm{A}}$ & $20.37 \pm 0.16^{\mathrm{B}}$ & $21.96 \pm 0.22^{\mathrm{A}}$ & $21.38 \pm 0.14^{\mathrm{C}}$ & $21.59 \pm 0.28^{\mathrm{A}}$ \\
\hline 21 & $22.15 \pm 0.11^{\mathrm{A}}$ & $20.63 \pm 0.14^{\mathrm{B}}$ & $20.72 \pm 0.21^{\mathrm{B}}$ & $19.76 \pm 0.10^{\mathrm{AB}}$ & $19.26 \pm 0.12^{\mathrm{AB}}$ & $19.06 \pm 0.17^{\mathrm{C}}$ & $19.69 \pm 0.15^{\mathrm{B}}$ & $20.18 \pm 0.12^{\mathrm{D}}$ & $21.04 \pm 0.38^{\mathrm{A}}$ \\
\hline 28 & $20.78 \pm 0.37^{\mathrm{AB}}$ & $18.81 \pm 0.33^{\mathrm{C}}$ & $21.76 \pm 0.13^{\mathrm{AB}}$ & $20.53 \pm 0.19^{\mathrm{A}}$ & $17.10 \pm 0.68^{\mathrm{B}}$ & $18.10 \pm 0.20^{\mathrm{CD}}$ & $18.81 \pm 0.33^{\text {В }}$ & $21.76 \pm 0.13^{\mathrm{BC}}$ & $20.87 \pm 0.29^{\mathrm{A}}$ \\
\hline 35 & $17.71 \pm 0.85^{\mathrm{B}}$ & $18.85 \pm 0.15^{\mathrm{C}}$ & $18.61 \pm 0.03^{\mathrm{C}}$ & $18.45 \pm 0.03^{\mathrm{B}}$ & $17.07 \pm 0.10^{\mathrm{B}}$ & $17.56 \pm 0.25^{\mathrm{D}}$ & $19.08 \pm 0.27^{\mathrm{B}}$ & $17.63 \pm 0.18^{\mathrm{E}}$ & $19.03 \pm 0.19^{\mathrm{B}}$ \\
\hline \multicolumn{10}{|l|}{$b^{*}$} \\
\hline 1 & $10.59 \pm 0.31^{\mathrm{A}}$ & $10.02 \pm 0.08^{\mathrm{B}}$ & $10.29 \pm 0.17^{\mathrm{B}}$ & $10.50 \pm 0.21^{\mathrm{AB}}$ & $11.87 \pm 0.05^{\mathrm{B}}$ & $11.93 \pm 0.04^{\mathrm{A}}$ & $11.49 \pm 0.27^{\mathrm{A}}$ & $11.21 \pm 0.14^{\mathrm{B}}$ & $10.19 \pm 0.14^{\mathrm{A}}$ \\
\hline 7 & $10.70 \pm 0.21^{\mathrm{A}}$ & $10.17 \pm 0.12^{\mathrm{B}}$ & $10.38 \pm 0.12^{\mathrm{B}}$ & $10.65 \pm 0.30^{\mathrm{AB}}$ & $11.80 \pm 0.09^{\mathrm{B}}$ & $11.82 \pm 0.13^{\mathrm{A}}$ & $11.32 \pm 0.11^{\mathrm{A}}$ & $10.99 \pm 0.02^{\mathrm{B}}$ & $10.74 \pm 0.09^{\mathrm{A}}$ \\
\hline 14 & $11.72 \pm 0.57^{\mathrm{A}}$ & $9.76 \pm 0.32^{\mathrm{B}}$ & $10.30 \pm 0.23^{\mathrm{B}}$ & $10.41 \pm 0.17^{\mathrm{AB}}$ & $11.64 \pm 0.11^{\mathrm{B}}$ & $11.89 \pm 0.18^{\mathrm{A}}$ & $11.21 \pm 0.19^{\mathrm{A}}$ & $11.09 \pm 0.11^{\mathrm{B}}$ & $10.83 \pm 0.14^{\mathrm{A}}$ \\
\hline 21 & $11.77 \pm 0.24^{\mathrm{A}}$ & $10.36 \pm 0.20^{\mathrm{B}}$ & $10.78 \pm 0.11^{\mathrm{B}}$ & $11.49 \pm 0.19^{\mathrm{A}}$ & $12.23 \pm 0.10^{\mathrm{AB}}$ & $12.33 \pm 0.10^{\mathrm{A}}$ & $11.43 \pm 0.12^{\mathrm{A}}$ & $11.36 \pm 0.10^{\mathrm{B}}$ & $11.21 \pm 0.33^{\mathrm{A}}$ \\
\hline 28 & $11.20 \pm 0.25^{\mathrm{A}}$ & $11.78 \pm 0.16^{\mathrm{A}}$ & $12.21 \pm 0.10^{\mathrm{A}}$ & $9.86 \pm 0.07^{\mathrm{B}}$ & $11.76 \pm 0.16^{\mathrm{B}}$ & $12.03 \pm 0.10^{\mathrm{A}}$ & $11.78 \pm 0.16^{\mathrm{A}}$ & $12.21 \pm 0.10^{\mathrm{A}}$ & $11.11 \pm 0.17^{\mathrm{A}}$ \\
\hline 35 & $9.59 \pm 1.02^{\mathrm{A}}$ & $10.33 \pm 0.21^{\mathrm{B}}$ & $10.65 \pm 0.13^{\text {В }}$ & $11.37 \pm 0.07^{\mathrm{A}}$ & $12.77 \pm 0.13^{\mathrm{A}}$ & $12.11 \pm 0.38^{\mathrm{A}}$ & $10.70 \pm 0.16^{\mathrm{A}}$ & $11.02 \pm 0.12^{\mathrm{B}}$ & $10.97 \pm 0.11^{\mathrm{A}}$ \\
\hline \multicolumn{10}{|l|}{$\Delta \mathrm{E}^{*}$} \\
\hline 1 & $50.06 \pm 0.20^{\mathrm{A}}$ & $49.09 \pm 0.31^{\mathrm{AB}}$ & $50.83 \pm 0.43^{\mathrm{AB}}$ & $46.01 \pm 0.63^{\mathrm{A}}$ & $45.09 \pm 0.24^{\mathrm{A}}$ & $49.09 \pm 0.31^{\mathrm{B}}$ & $48.96 \pm 0.53^{\mathrm{A}}$ & $50.06 \pm 0.20^{\mathrm{AB}}$ & $45.21 \pm 0.13^{\mathrm{A}}$ \\
\hline 7 & $51.60 \pm 0.38^{A}$ & $48.48 \pm 0.39^{\mathrm{AB}}$ & $50.92 \pm 0.09^{\mathrm{AB}}$ & $45.82 \pm 0.42^{\mathrm{A}}$ & $46.51 \pm 1.54^{\mathrm{A}}$ & $50.92 \pm 0.09^{\mathrm{AB}}$ & $48.76 \pm 0.36^{\mathrm{A}}$ & $49.55 \pm 0.49^{\mathrm{ABC}}$ & $45.99 \pm 0.20^{\mathrm{A}}$ \\
\hline 14 & $50.45 \pm 1.62^{\mathrm{A}}$ & $48.79 \pm 1.26^{\mathrm{AB}}$ & $50.08 \pm 0.71^{\mathrm{AB}}$ & $46.55 \pm 0.21^{\mathrm{A}}$ & $45.60 \pm 0.01^{\mathrm{A}}$ & $50.08 \pm 0.71^{\mathrm{AB}}$ & $47.98 \pm 0.53^{\mathrm{AB}}$ & $49.57 \pm 0.67^{\mathrm{ABC}}$ & $46.90 \pm 0.52^{\mathrm{A}}$ \\
\hline 21 & $50.61 \pm 0.80^{\mathrm{A}}$ & $49.92 \pm 0.69^{\mathrm{AB}}$ & $49.48 \pm 0.27^{\mathrm{B}}$ & $47.09 \pm 0.66^{\mathrm{A}}$ & $45.23 \pm 0.16^{\mathrm{A}}$ & $49.48 \pm 0.27^{\mathrm{AB}}$ & $46.30 \pm 0.11^{\mathrm{B}}$ & $48.42 \pm 0.78^{\mathrm{BC}}$ & $46.58 \pm 1.07^{\mathrm{A}}$ \\
\hline 28 & $50.94 \pm 0.27^{\mathrm{A}}$ & $49.94 \pm 0.36^{\mathrm{B}}$ & $52.43 \pm 0.33^{\mathrm{B}}$ & $45.98 \pm 0.35^{\mathrm{A}}$ & $45.09 \pm 1.44^{\mathrm{A}}$ & $44.40 \pm 0.39^{\mathrm{C}}$ & $46.94 \pm 0.36^{\mathrm{AB}}$ & $52.43 \pm 0.33^{\mathrm{A}}$ & $45.93 \pm 0.50^{\mathrm{A}}$ \\
\hline 35 & $46.97 \pm 2.27^{\mathrm{A}}$ & $51.98 \pm 0.45^{\mathrm{A}}$ & $49.74 \pm 0.42^{\mathrm{B}}$ & $45.61 \pm 0.20^{\mathrm{A}}$ & $44.65 \pm 0.33^{\mathrm{A}}$ & $51.98 \pm 0.45^{\mathrm{A}}$ & $46.16 \pm 0.14^{\mathrm{B}}$ & $46.44 \pm 0.43^{\mathrm{C}}$ & $45.55 \pm 0.28^{\mathrm{A}}$ \\
\hline
\end{tabular}

${ }^{*}$ Mean and standard deviation; on the table, the same letters in lines and columns Express no difference statistically $(p>0.01)$, different letters express a difference statistically ( $\left.\mathrm{p}<0.05\right)$. 
According to the statistical analysis, storage time, coating type and storage time coating type interaction were significant statistically on $\mathrm{pH}$ values of bologna-type sausage which applied antimicrobial PASP coatings $(\mathrm{p}<0.01)$. During the storage time, $\mathrm{N}$-WPI was the most effective coating type. PS-WPI, SB-WPI, and control coatings had no significant effect on $\mathrm{pH}$ value $(\mathrm{p}<0.01)$. When the storage period was examined, it is seen that the highest $\mathrm{pH}$ value $\left(\mathrm{pH}\right.$ 6.47) was reached on the $7^{\text {th }}$ day of storage. This was followed by $\mathrm{pH}$ values at 1, 14, 28 and 21 days, respectively. The lowest $\mathrm{pH}$ value ( $\mathrm{pH}$ 6.24) was obtained on the $35^{\text {th }}$ day of storage time $(\mathrm{p}<0.01)$. It was seen that the $\mathrm{pH}$ value of the samples exceeds the $\mathrm{pH}$ value (6.40) given in TSE 979, especially in the samples with WPI coating containing thyme essential oil and sodium benzoate. Towards the last storage days, all antimicrobial WPI-treated bologna-type sausage samples showed a reduction in $\mathrm{pH}$ values. This situation can be attributed to the L. innocua counts increase, especially since the $14^{\text {th }}$ and $21^{\text {st }}$ days of storage.

During the storage period, aw values of samples were increased (Table 2). These results were not significant statistically for N-WPI, however, were found to be significant for other antimicrobial WPI coatings $(\mathrm{p}<0.01)$. According to the statistical analysis, the interaction of the storage time, coating type and storage time coating type were significant statistically on the aw values of antimicrobial WPI-coated bologna-type sausages $(\mathrm{p}<0.01)$. During storage time, the lowest aw value (0.945) was obtained with WPI coating containing thyme essential oil. This value was followed by WPI coating species containing of coriander, allspice, basil, rosemary essential oils ( $4 \% \mathrm{w} / \mathrm{v})$ and sodium benzoate, potassium sorbate $(4 \% \mathrm{w} / \mathrm{v})$ and nisin $(10.000 \mathrm{IU})$ at equal levels. The highest aw value (0.952) was detected in the control WPI coatings $(\mathrm{p}<0.01)$. When accordingly, the lowest aw value (0.941) was observed on the first day of storage and the highest $a_{w}$ value $(0.954)$ on the $35^{\text {th }}$ day of storage $(\mathrm{p}<0.01)$. When examined of the effect of storage time on aw values, aw values on days 1, 7 and 14 were statistically significant. The differences between $a_{w}$ values on the other days of storage were not significant statistically. Accordingly, the lowest $\mathrm{a}_{\mathrm{w}}$ value (0.941) was observed on the first day of storage time and the highest aw value (0.954) on the $35^{\text {th }}$ day of storage $(\mathrm{p}<0.01)$. When evaluated in general terms, $a_{w}$ values of Bologna-type sausage samples covered with antimicrobial WIP coatings were between $0.857-0.993$ values who stated by Özay et al. (1993).

As shown in Table 3, the application of the antimicrobial WIP coatings was not causing significant changes in the color properties of the Bologna-type sausage samples $(\mathrm{p}<0.01)$. When the coating type and storage time were statistically significant, coating type and the storage time interaction was statistically insignificant on $L$ values $(\mathrm{p}<0.01)$. Accordingly, the highest $L$ value (57.80) was obtained with B-WPI, and the lowest average $L$ value (52.43) was obtained with C-WPI. In addition, the highest $L$ value (55.68) was found on day 7 of storage, while the lowest $L$ value (55.09) was found on day 28 of storage $(\mathrm{p}<0.01)$. Similarly, Jo et al. (2001) reported that sausages containing chitosan had higher $L^{*}$ values than the control samples during storage. Additionally, Giatrakou et al. (2010) found that the addition of chitosan to a ready-to-eat chicken product increased the $L^{*}$ value. Coating type, storage time, coating type and storage time interaction were statistically significant on the values of $a(\mathrm{p}<0.01)$. Accordingly, the highest $a$ value (21.50) was obtained by Control-WPI, while the lowest $a$ value (19.08 and 19.20) was determined in WPI coatings containing coriander and basil essential oil. During the storage period, when $a$ value was examined, the highest value was determined on the 7 th day of storage (21.97) while the lowest value $(18.22)$ was obtained on the 35 th day $(\mathrm{p}<0.01)$. Similar to the results of our study, Georgantelis et al. (2007) determined that the combination of chitosan and rosemary extract acted synergistically to improve the redness of beef burgers, during frozen storage, while individually chitosan or rosemary extract improved the color stability relative to control. On the $b$ values, the coating type, storage time and coating type and storage time interaction were found to be statistically significant $(\mathrm{p}<0.01)$. Accordingly, the highest $b$ value (12.02) was determined in the samples of bologna-type sausage with R-WPI coating. The lowest $b$ value (10.40) was found on the samples with T-WPI coatings. The highest $b$ values were determined with 11.55 on the 28 th day of storage, and the lowest $b$ values were found with 10.90 on the 1 st day of storage ( $\mathrm{p}<0.01)$. Similarly, Youn et al. (1999) and Jo et al. (2001) reported that the addition of chitosan increased the $b^{\star}$ value of sausages during storage, suggesting that the natural color of chitosan affected the surface color of the sausage. Coating type, storage time and coating type * storage time interaction were found statistically significant on the values of $\Delta \mathrm{E}(\mathrm{p}<0.01)$. Accordingly, the highest $\Delta \mathrm{E}$ values (50.59 and 50.10) were obtained with Control-WPI and C-WPI coatings, while the lowest E parameter value (45.36) was obtained with B-WPI coatings. When the highest $\Delta \mathrm{E}$ value (48.73) was determined on the 7 th day of storage, the lowest $\Delta \mathrm{E}$ value (47.68) was determined on the 35 th day of storage $(\mathrm{p}<0.01)$.

\subsection{Sensory evaluation}

Sensory analysis evaluated by an expert jury (food engineers) for each bologna-type sausage samples applied antimicrobial coatings and general appearance, color, smell, texture, taste and general product appreciation related scores of samples were shown in Table 4. Sensory analysis was performed using a 9-point scale. As seen in Table 4, coating type and storage time were significant statistical effects on the sensory scores of Bologna-type sausage samples covered WIP coated containing different antimicrobial agent $(\mathrm{p}<0.05)$. It was determined that average of the highest overall appearance score (8.28) was obtained with T-WPI coatings. Samples with the lowest overall appearance score (6.95) were determined by B-WPI coatings. When the effects of storage time on overall mean scores were examined, the samples of with antimicrobial WPI coatings with the highest overall appearance averages (8.04) were determined on 14th day of storage time. The lowest mean overall score (7.30) was obtained on the 28 th day of storage $(p<0.05)$. When samples were evaluated in terms of color properties, the highest color scores average value (8.34) was determined by C-WPI coatings. The lowest color scores average value (6.59) was obtained with R-WPI coatings. Coating type and storage time were found to be significant statistically, however, coating type and storage time interaction were insignificant on the odor scores average $(\mathrm{p}<0.05)$. In this context, the highest odor scores average values (8.46) was N-WPI coated samples while the lowest odor score (7.83) was found to be with B-WPI coatings. When a general 
Table 4. Sensory properties of Bologna-type sausage slices coated with WPI solution containing different antimicrobials during storage.

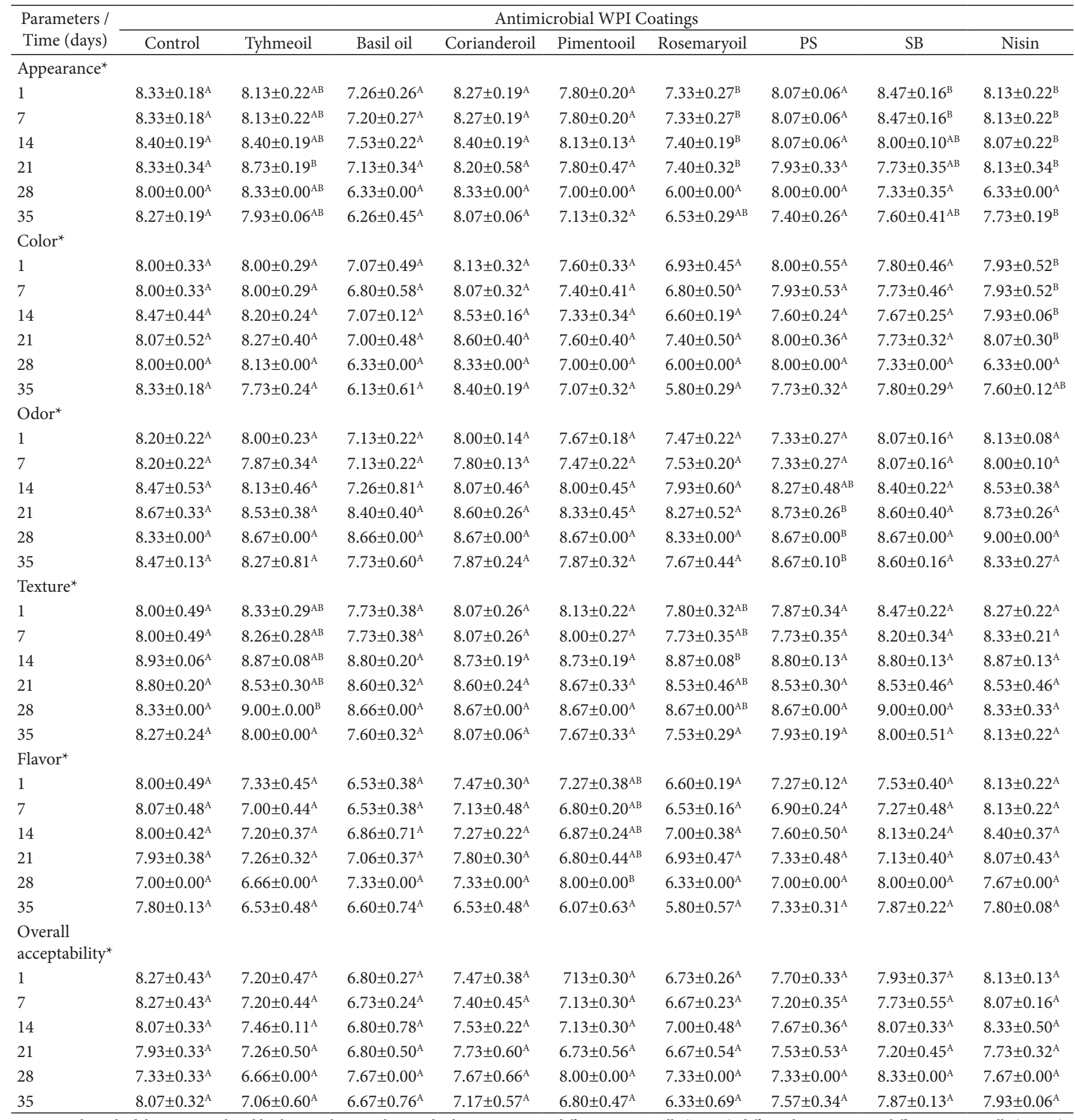

${ }^{\star}$ Mean and standard deviation; on the table, the same letters in lines and columns express no difference statistically $(p>0.05)$, different letters express a difference statistically ( $\left.<<0.05\right)$.

evaluation was made of texture scores of samples, storage time was significant statistically. However, coating type and coating type and storage time interaction were found to be insignificant $(\mathrm{p}<0.05)$. The highest texture scores averaged $(8.82)$ was obtained at 14th day of storage time while the lowest scores averaged was found to be 7.93 on the 35th day of the storage time. When the taste scores of the samples were examined, it was statistically significant for the taste point averages of the samples of bologna type-sausage with WPI coatings containing only allspice essential oil, while it was found insignificant for all other coated samples $(\mathrm{p}<0.05)$. Coating type and storage time were significant on the average taste scores of samples while the interaction of the coating type and storage time were not found to be significant $(\mathrm{p}<0.05)$. The highest taste scores average (8.03) was found to be N-WPI coatings samples groups. The lowest taste score was determined as 6.53 for R-WPI coated samples. 
When the relationship between the storage period and samples with antimicrobial WPI coating were examined, the mean value of the highest taste scores average (7.53) was reached on the 14 th day of storage. However, the lowest taste scores average (6.97) was obtained on the 35th day of storage time $(\mathrm{p}<0.05)$. As another sensory property, the effect of the coating on the overall product appreciation was examined, highest average score was determined to be 7.99 on Control-WPI coated samples. The lowest overall product average value of samples was found to be 6.79 on R-WPI coatings. As the conclusion, the results of sensory evaluation differ in cases of antimicrobial WPI coated Bologna-type sausage samples. It was possible to say that the use of WPI coatings containing essential oils is not very favorable for use in bologna-type sausage, whereas the application of Nisin containing WPI coatings is the most admired coating types. According to the results of sensory analysis obtained, Nisin-WPI coatings may be recommended for use in ready-to-eat meat products. Similarly, in a prior published study, Lekjing (2016) investigated the effects of chitosan-based coating incorporated with clove oil on cooked pork sausage samples in refrigerated storage. The results of this study indicate that pork sausages' shelf life can be extended by approximately 5 days, using chitosan alone or in combination with clove oil. Aside from the odor, the combination with clove oil treatment consistently performed better than chitosan alone in all other sensory attributes, throughout the entire storage period.

\section{Conclusion}

As a result of this study, it has been determined that coatings containing potassium sorbate and sodium benzoate were the most effective antimicrobial coatings against $L$. innocua and coatings containing thyme essential oil were the most sensory coating types. However, the inactivation achieved with potassium sorbate and sodium benzoate coatings was carried out by adding the active antimicrobials to the coating at a very high value of $4 \%(\mathrm{w} / \mathrm{v})$. For this reason, it will be more appropriate to use potassium sorbate and sodium benzoate in combination with essential oils and Nisin that have been found to have antimicrobial activity on L. innocua, taking advantage of the barrier technology. Thus, that the amounts of these antimicrobials in coating solutions can be reduced. It is believed that in the case of edible films and coatings, for the antimicrobial efficacy of other legally permissible preservatives is evaluated, that the results of the study are beneficial and will lead to future work. Packages containing antimicrobial preservatives, such as each antimicrobial agent used in this study, will be useful in preventing microbiological deterioration, ensuring food safety and prolonging the shelf life of the product during storage in bologna-type sausage samples. In this context, antimicrobial edible coatings are highly promising for protection and development of product quality in the future.

\section{Acknowledgements}

This study was supported by Cukurova University Scientific Research Projects Coordination Unit (Project Number: ZF2011D24).

\section{References}

Amaral, G. V., Silva, E. K., Costa, A. L. R., Alvarenga, V. O., Cavalcanti, R. N., Esmerino, E. A., Guimarães, J. T., Freitas, M. Q., Sant'Ana, A. S., Cunha, R. L., Moraes, J., Silva, M. C., Meireles, M. A. A., \& Cruz, A. G. (2018). Whey-grape juice drink processed by supercritical carbon dioxide technology: physical properties and sensory acceptance. Lebensmittel-Wissenschaft + Technologie, 92, 80-86. http://dx.doi. org/10.1016/j.lwt.2018.02.005.

Andrews, W. H., June, G. A., Sherrod, P. S., Hammack, T. S., \& Amaguana, R. M. (1995). FDA Bacteriological analytical manual. Gaithersburg: AOAC International.

Appendini, P., \& Hotchkiss, J. H. (2002). Review of antimicrobial food packaging. Innovative Food Science \& Emerging Technologies, 3(2), 113-126. http://dx.doi.org/10.1016/S1466-8564(02)00012-7.

Bahram, S., Rezaei, M., Soltani, M., Kamali, A., Ojagh, S. M., \& Abdollahi, M. (2013). Whey protein concentrate edible film activated with cinnamon essential oil. Journal of Food Processing and Preservation, 38(3), 1251-1258. http://dx.doi.org/10.1111/jfpp.12086.

Barancelli, G. V., Camargo, T. M., Gagliardi, N. G., Porto, E., Souza, R. A., Campioni, F., Falcão, J. P., Hofer, E., Cruz, A. G., \& Oliveira, C. A. (2014). Pulsed-Field Gel Electrophoresis characterization of Listeria monocytogenes isolates from cheese manufacturing plants in São Paulo, Brazil. International Journal of Food Microbiology, 173, 21-29. http://dx.doi.org/10.1016/j.ijfoodmicro.2013.12.018. PMid:24412413.

Buchrieser, C., Rusniok, C., Kunst, F., Cossart, P., \& Glaser, P. (2003). Comparison of the genome sequences of Listeria monocytogenes and Listeria innocua: clues for evolution and pathogenicity. FEMS Immunology and Medical Microbiology, 35(3), 207-213. http://dx.doi. org/10.1016/S0928-8244(02)00448-0. PMid:12648839.

Cağri, A., Ustunol, Z., \& Ryser, E. T. (2002). Inhibition of three pathogens on bologna and summer sausage using antimicrobial edible films. Journal of Food Science, 67(6), 2317-2324. http://dx.doi. $\operatorname{org} / 10.1111 /$ j.1365-2621.2002.tb09547.x.

Cao-Hoang, L., Chaine, A., Gregoire, L., \& Wache, Y. (2010). Potential of nisin-incorporated sodium caseinate films to control Listeria in artificially contaminated cheese. Food Microbiology, 27(7), 940-944. http://dx.doi.org/10.1016/j.fm.2010.05.025. PMid:20688236.

Cooksey, K. (2005). Effectiveness of antimicrobial food packaging materials. Food Addition Contaminant, 22(10), 980-987. http:// dx.doi.org/10.1080/02652030500246164. PMid:16227182.

Coutinho, N. M., Silveira, M. R., Fernandes, L. M., Moraes, J., Pimentel, T. C., Freitas, M. Q., Silva, M. C., Raices, R. S. L., Ranadheera, C. S., Borges, F. O., Neto, R. P. C., Tavares, M. I. B., Fernandes, F. A. N., Fonteles, T. V., Nazzaro, F., Rodrigues, S., \& Cruz, A. G. (2019). Processing chocolate milk drink by low-pressure cold plasma technology. Food Chemistry, 278, 276-283. http://dx.doi.org/10.1016/j. foodchem.2018.11.061. PMid:30583374.

Davies, E. A., Milne, C. F., Bevis, H. E., Potter, R. W., Harris, J. M., Williams, G. C., Thomas, L. V., \& Delves-Broughton, J. (1999). Effective use of nisin to control lactic acid bacterial spoilage in vacuum-packed bologna-type sausage. Journal of Food Protection, 62(9), 1004-1010. http://dx.doi.org/10.4315/0362-028X-62.9.1004. PMid:10492474.

Erginkaya, Z., Kalkan, S., \& Ünal, E. (2014, November 14). Use of antimicrobial edible films and coatings as packaging materials for food safety. In A. Malik, Z. Erginkaya, S. Ahmad, \& H. Erten, Food Processing: Strategies for Quality Assessment (2014 ed., pp. 261-295). New York: Springer. 
Fernandes, M. S., Cruz, A. G., Arroyo, D. M. D., José de Assis, F. F., Cristianini, M., \& Sant'Ana, A. S. (2013). On the behavior of Listeria innocua and Lactobacillus acidophilus co-inoculated in a dairy dessert and the potential impacts on food safety and product's functionality. Food Control, 34(2), 331-335. http://dx.doi.org/10.1016/j. foodcont.2013.04.040.

Food and Drug Administration - FDA. (2006). Substances generally recognized as safe. Code of Federal Regulations, Title 21, Parts 182 and 184. Washington: Office of the Federal Register, U.S. Government Printing Office.

Georgantelis, D., Blekas, G., Katikou, P., Ambrosiadis, I., \& Fletouris, D. J. (2007). Effect of rosemary extract, chitosan and $\alpha$-tocopherol on lipid oxidation and colour stability during frozen storage of beef burgers. Meat Science, 75(2), 256-264. http://dx.doi.org/10.1016/j. meatsci.2006.07.018. PMid:22063657.

Giatrakou, V., Ntzimani, A., \& Savvaidis, I. N. (2010). Effect of chitosan and thyme oil on a ready to cook chicken product. Food Microbiology, 27(1), 132-136. http://dx.doi.org/10.1016/j.fm.2009.09.005. PMid:19913703.

Glass, K. A., Veesenmeyer, J., McDonnell, L. M., Eimerman, P., \& Johnson, E. A. (2004). Control of Listeria monocytogenes in wiener and turkey slurries by combinations of antimicrobials. Journal of Food Protection, 67, 55.

Glass, K., Preston, D., \& Veesenmeyer, J. (2007). Inhibition of Listeria monocytogenes in turkey and pork-beef bologna by combinations of sorbates, benzoate and propionate. Journal of Food Protection, 70(1), 214-217. http://dx.doi.org/10.4315/0362-028X-70.1.214. PMid:17265884.

Horita, C. N., Esmerino, E. A., Vidal, V. A. S., Farah, J. S., Amaral, G. V., Bolini, H. M. A., Cruz, A. G., \& Pollonio, M. A. R. (2017). Sensory profiling of low sodium frankfurter containing garlic products: Adequacy of Polarized Projective Mapping compared with trained panel. Meat Science, 131, 90-98. http://dx.doi.org/10.1016/j. meatsci.2017.05.002. PMid:28500963.

Jo, C., Lee, J. W., Lee, K. H., \& Byun, M. W. (2001). Quality properties of pork sausage prepared with water-soluble chitosan oligomer. Meat Science, 59(4), 369-375. http://dx.doi.org/10.1016/S03091740(01)00089-4. PMid:22062961.

Karagöz, Z., \& Candoğan, K. (2007). Et teknolojisinde antimikrobiyel ambalajlama. Gida, 32, 113-122.

Kim, S. A., Park, S. H., Knueven, C., Basel, R., \& Ricke, S. C. (2018). A decontamination approach using a combination of bisulfate of soda and peracetic acid against Listeria innocua inoculated on whole apples. Food Control, 84, 106-110. http://dx.doi.org/10.1016/j. foodcont.2017.07.036.

Ku, K. J., Hong, Y. H., \& Song, K. B. (2008). Mechanical properties of a Gelidiumcorneum edible films containing catechin and its application in sausages. Journal of Food Science, 73(3), 217-221. http://dx.doi. org/10.1111/j.1750-3841.2008.00700.x. PMid:18387102.

Lekjing, S. (2016). A chitosan-based coating with or without clove oil extends the shelf life of cooked pork sausages in refrigerated storage. Meat Science, 111, 192-197. http://dx.doi.org/10.1016/j. meatsci.2015.10.003. PMid:26473294.

Murray, M., \& Richard, J. A. (1997). Comparative study of the antilisterial activity of nisin $\mathrm{A}$ and pediocinAcH in fresh ground pork stored aerobically at $5{ }^{\circ} \mathrm{C}$. Journal of Food Protection, 60(12), 1534-1540. http://dx.doi.org/10.4315/0362-028X-60.12.1534. PMid:31207757.

Odila Pereira, J., Soares, J., Monteiro, M., Gomes, A., \& Pintado, M. (2018). Impact of whey protein coating incorporated with Bifidobacterium and Lactobacillus on sliced ham properties. Meat Science, 139, 125133. http://dx.doi.org/10.1016/j.meatsci.2018.01.016. PMid:29413672.
Ozay, G., Pala, M., \& Sayg1, B. (1993). Bazı gıdaların su aktivitesi $\left(\mathrm{a}_{\mathrm{w}}\right)$ yönünden incelenmesi. Gida, 18, 377-383.

Ozdemir, M., \& Floros, J. D. (2008). Optimization of edible whey protein films containing preservatives for water vapor permeability, water solubility and sensory characteristics. Journal of Food Engineering, 86(2), 215-224. http://dx.doi.org/10.1016/j.jfoodeng.2007.09.028.

Pietrysiak, E., \& Ganjyal, G. M. (2018). Apple peel morphology and attachment of Listeria innocua through aqueous environment as shown by scanning electron microscopy. Food Control, 92, 362-369. http://dx.doi.org/10.1016/j.foodcont.2018.04.049.

Pires, C., Ramos, C., Teixeira, G., Batista, I., Mendes, R., Numes, L., \& Marques, A. (2011). Characterization of biodegradable films prepared with hake proteins and thyme oil. Journal of Food Engineering, 105(3), 422-428. http://dx.doi.org/10.1016/j.jfoodeng.2011.02.036.

Pranoto, Y., Salokhe, V. M., \& Rakshit, S. K. (2005). Physical and antibacterial properties of alginate-based edible film incorporated with garlic oil. Food Research International, 38(3), 267-272. http:// dx.doi.org/10.1016/j.foodres.2004.04.009.

Ramos, O. L., Silva, S. I., Soares, J. C., Fernandes, J. C., Poças, M. F., Pintado, M. E., \& Malcata, F. X. (2012). Features and performance of edible films, obtained from whey protein isolate formulated with antimicrobial compounds. Food Research International, 45(1), 351361. http://dx.doi.org/10.1016/j.foodres.2011.09.016.

Royo, M., Fernandez-Pan, I., \& Mate, J. I. (2010). Antimicrobial effectiveness of oregano and sage essential oils incorporated in to whey protein films or cellulose-based filter paper. Journal of the Science of Food and Agriculture, 90(9), 1513-1519. http://dx.doi. org/10.1002/jsfa.3977. PMid:20549805.

Santos, B. A., Bastianello Campagnol, P. C., Cruz, A. G., Galvão, M. T. E. L., Monteiro, R. A., Wagner, R., \& Pollonio, M. A. R. (2015). Check all that apply and free listing to describe the sensory characteristics of low sodium dry fermented sausages: Comparison with trained panel. Food Research International, 76(Pt 3), 725-734. http://dx.doi. org/10.1016/j.foodres.2015.06.035. PMid:28455058.

Sarıkuş, G. (2006). Farklı antimikrobiyel maddeler içeren yenilebilir film üretimi ve kaşar peynirinin muhafazasında mikrobiyel inaktivasyona etkisi (MSc thesis). Süleyman Demirel University, Isparta, Turkey.

Sheridan, J. J., Duffy, G., McDowell, D. A., \& Blair, I. S. (1997). Development of a surface adhesion immunofluorescent technique for the rapid isolation of Listeria monocytogenes and Listeria innocua from meat. Journal of Applied Microbiology, 82(2), 225-232. http:// dx.doi.org/10.1111/j.1365-2672.1997.tb03577.x. PMid:12452598.

Silveira, M. R., Coutinho, N. M., Esmerino, E. A., Moraes, J., Fernandes, L. M., Pimentel, T. C., Freitas, M. Q., Silva, M. C., Raices, R. S. L., Senaka Ranadheera, C., Borges, F. O., Neto, R. P. C., Tavares, M. I. B., Fernandes, F. A. N., Fonteles, T. V., Nazzaro, F., Rodrigues, S., \& Cruz, A. G. (2019). Guava-flavored whey beverage processed by cold plasma technology: Bioactive compounds, fatty acid profile and volatile compounds. Food Chemistry, 279, 120-127. http://dx.doi. org/10.1016/j.foodchem.2018.11.128. PMid:30611471.

Suppakul, P., Miltz, J., Sonneveld, K., \& Bigger, S. W. (2003). Active packaging technologies with an emphasis on antimicrobial packaging and its applications. Journal of Food Science, 68(2), 408-420. http:// dx.doi.org/10.1111/j.1365-2621.2003.tb05687.x.

Tajkarimi, M. M., Ibrahim, S. A., \& Cliver, D. O. (2010). Antimicrobial herb and spice compounds in food. Food Control, 21(9), 1199-1218. http://dx.doi.org/10.1016/j.foodcont.2010.02.003.

Theivendran, S., Hettiarachchy, N. S., \& Johnson, M. G. (2006). Johnson M. Inhibition of Listeria monocytogenes by nisin combined with grape seed extract or green tea extract in soy protein film coated 
on turkey frankfurters. Journal of Food Science, 71(2), 39-44. http:// dx.doi.org/10.1111/j.1365-2621.2006.tb08905.x.

Tiryaki, G. G. (2008). Engelleme teknolojisinin sebzelerde patojen inaktivasyonu ve raf ömrü açısından etkilerinin araştırılması ( $\mathrm{PhD}$ Thesis). Ege University, İzmir, Turkey.

Tomé, E., Gibbs, P. A., \& Teixeira, P. C. (2008). Growth control of L.innocua 2030c on vacuum-packaged cold smoked salmon by lactic acid bacteria. International Journal of Food Microbiology, 121(3), 285-294. http://dx.doi.org/10.1016/j.ijfoodmicro.2007.11.015. PMid:18068846.

Vidal, V. A., Biachi, J. P., Paglarini, C. S., Pinton, M. B., Campagnol, P. C., Esmerino, E. A., Cruz, A. G., Morgano, M. A., \& Pollonio, M. A. R. (2019). Reducing 50\% sodium chloride in healthier jerked beef: An efficient design to ensure suitable stability, technological and sensory properties. Meat Science, 152, 49-57. http://dx.doi. org/10.1016/j.meatsci.2019.02.005. PMid:30802818.
Ye, M., Neetoo, H., \& Chen, H. (2008). Effectiveness of chitosan-coated plastic films intercorporating antimicrobials in inhibition of Listeria monocytogenes on cold-smoked salmon. International Journal of Food Microbiology, 127(3), 235-240. http://dx.doi.org/10.1016/j. ijfoodmicro.2008.07.012. PMid:18707789.

Youn, S. K., Park, S. M., Kim, Y. J., \& Ahn, D. H. (1999). Effect on storage property and quality in meat sausage by added chitosan. Journal of Chitin and Chitosan, 4, 189-195.

Zhu, M., Du, M., Cordray, J., \& Ahn, D. U. (2005). Control of Listeria monocytogenes contamination in ready-to-eat meat products. Comprehensive Reviews in Food Science and Food Safety, 4(2), 34-42. http://dx.doi.org/10.1111/j.1541-4337.2005.tb00071.x.

Zinoviadou, K. G., Koutsoumanis, K. P., \& Biliaderis, C. G (2009). Physico-chemical properties of whey protein isolate films containing oregano oil and their antimicrobial action against spoilage flora of fresh beef. Meat Science, 82(3), 338-345. http://dx.doi.org/10.1016/j. meatsci.2009.02.004. PMid:20416718. 